Published in final edited form as:

Curr Oncol Rep. ; 21(7): 56. doi:10.1007/s11912-019-0806-2.

\title{
Immunotherapy in Older Adults with Cancer
}

\author{
Richard Curtis Godby, MD11, Douglas B. Johnson, $\mathbf{M D}^{2}$, and Grant R. Williams, MD ${ }^{3,}{ }^{*}$ \\ ${ }^{1}$ Internal Medicine, University of Alabama at Birmingham, Birmingham, AL, United States \\ ${ }^{2}$ Division of Hematology/Oncology, Vanderbilt University Medical Center, Nashville, TN, United \\ States \\ ${ }^{3}$ Division of Hematology/Oncology, University of Alabama at Birmingham, Birmingham, AL, United \\ States
}

\begin{abstract}
Purpose of review: The purpose of this review was to examine the latest research and data on the use of immunotherapy in older adults with cancer in order to identify key gaps in the literature for future research.
\end{abstract}

Recent findings: Immune checkpoint inhibitors are gaining approval and being incorporated into routine clinical use for numerous malignancies across age groups due to their overall efficacy and favorable side effect profiles.

Summary: Although immune checkpoint inhibitors appear both safe and effective in older adults, deliberate study of immunotherapies in older adults is highly warranted given the paucity of data in a population with unique immunobiology that comprises the majority of the cancer population worldwide.

\section{Keywords}

Immunotherapy; Geriatric Oncology; Aged; Immunosenescence; Immune Checkpoint Inhibitors; Clinical Trial Representation

\section{Introduction}

Advancing age remains the single most significant risk factor for most cancers, and adults 65 years or older are most susceptible. As the US population continues to age, the number of adults over age 65 with cancer is expected to double by 2030 with projections that nearly $70 \%$ of all new cancer diagnoses will be in older adult (1). Despite these dramatically increasing numbers, older adults with cancer are routinely underrepresented in clinical research; less than $25 \%$ of patients enrolled in NCI-cooperative group clinical trials are $65-$ 74 years and $<10 \%$ are over 75 years $(2,3)$. This lack of representation has led to many

*Corresponding Author: Grant R. Williams, MD, Assistant Professor, Divisions of Hematology/Oncology \& Gerontology, Geriatrics, and Palliative Care, Institute of Cancer Outcomes and Survivorship, O'Neal Comprehensive Cancer Center at UAB, University of Alabama at Birmingham, 1600 7th Avenue South, Lowder 500, Birmingham, AL 35233, grwilliams@ uabmc.edu, Phone: 001-205-638-7000, Fax: 001-205-638-2121, USA. 
significant knowledge gaps that limit our ability to optimize care for this growing and vulnerable population.

The use of chronological age in evaluating and treating older adults is insufficient due to the significant variability in physiologic age and outcomes in patients of identical chronological age $(4,5)$. Impaired functional reserve, comorbid conditions, and organ dysfunction frequently occur in older patients and have significant implications for cancer treatment selection and decision-making (6). Additionally, the high prevalence of low muscle mass, otherwise known as sarcopenia, poises an additional challenge in caring for older adults with cancer (7). Studies have shown that age and performance status assessments are not sufficient to predict efficacy and toxicity from therapy in older adults with cancer $(8,9)$. More comprehensive examinations, such as the geriatric assessment, are recommended in the evaluation of older adults considering chemotherapy to better assess the risk/benefit of treatment decisions (3). These global and comprehensive assessments can aid in developing personalized cancer treatment plans (10).

Over the past several years, new treatment modalities utilizing immunotherapies have radically altered treatment algorithms for numerous and diverse cancers. Response rates to immunotherapies range from $20 \%$ to $>80 \%$ depending on the cancer type, and unlike responses to traditional cytotoxic therapies, many of the responses can last for years. Moreover, these agents are often well-tolerated compared to traditional chemotherapy with lower rates of severe toxicities. The attractive durable response rates and toxicity profiles of new immunotherapy treatments has led to a new set of treatment decisions surrounding older patients with cancer, and those who might have been considered ineligible for cytotoxic chemotherapy are often treated with immunotherapies, although the overall tolerability and responses to immunotherapy in this population is poorly understood (11). Aging has various well-characterized effects on global immune function (termed "Immunosenescence"), including decreased thymic $\mathrm{T}$ cell production, shifts in $\mathrm{T}$ cell subsets, and deficits in $\mathrm{T}$ cell function, and how these age-related changes impact the response to immunotherapy remains largely unknown (12-15).

The purpose of this review was to examine the latest research and data on the use of immunotherapy in older adults with cancer and to identify key gaps in the literature for future research.

\section{Immune Checkpoint Inhibitors}

Agents that activate the immune system to target cancer have made major inroads in improving outcomes in patients with metastatic cancer over the last several years. Specifically, immune checkpoint inhibitors (ICIs) have now received regulatory approval in 15 different cancer types and produce long-term responses in a subset of patients. ICIs have emerged as a fifth pillar of cancer therapeutics, along with surgery, radiation, cytotoxic chemotherapy, and molecularly targeted therapy.

Three distinct classes of ICI have received regulatory approval: agents targeting cytotoxic T lymphocyte antigen-4 (CTLA-4), programmed death-1 receptor (PD-1), and programmed 
death-1 ligand (PD-L1). T cell co-stimulation, as occurs in interactions between dendritic cells and $\mathrm{T}$ cells in draining lymph nodes, occurs when $\mathrm{CD} 28$, a $\mathrm{T}$ cell receptor, binds its ligand B7 on dendritic cells. CTLA-4 blunts this co-stimulation by binding B7 at higher affinity, thus dampening the immune response. Blocking CTLA-4 (eg, with the monoclonal antibody ipilimumab) inhibits this negative regulator, functionally "removing the brakes" on T cell co-stimulation. By contrast, PD-1 and PD-L1 function more "distally" in the immune response, at the level of effector function. PD-1, present on T cells, binds its ligands PD-L1 and PD-L2 (which are expressed in many tumors and other sites of inflammation), producing T cell exhaustion. Inhibition of either PD-1 or PD-L1 with monoclonal antibodies targeting these molecules produces a more effective immune response, leading to greater activity than anti-CTLA-4 across cancers.

\section{PD-1 Inhibitors}

There are currently three FDA-approved PD-1 inhibitors, nivolumab (Opdivo®, BristolMyers Squibb), pembrolizumab (Keytruda ${ }^{\circledR}$, Merck), and cemiplimab (Libtayo®, Regeneron) with additional agents under development. Nivolumab and pembrolizumab were each approved in 2014 for metastatic melanoma but have since gone on to garner multiple indications (Table 1), with active investigations into additional uses underway. In 2017, pembrolizumab became the first immunotherapy approved based upon a tumor's genetic aberrations rather than its primary origin of development (for microsatellite instable cancers). These agents are monoclonal antibodies directed against PD-1 and require intravenous infusion over 30 minutes, with nivolumab administered every two to four weeks and pembrolizumab administered every three weeks.

PD-1 is a transmembrane receptor located on T-cells that typically binds to the PD-L1 located on antigen presenting cells (APCs) and prevents autoimmune disease by facilitating self-tolerance. As various tumor cells express PD-L1 to achieve immune evasion, the PD-1 inhibitors promote anti-tumor activity via disinhibition of T-cells and other immunomodulatory mechanisms, but simultaneously facilitate side effects known as immune-related adverse events (irAEs) by lowering the threshold of self-tolerance (Table 2). Compared with anti-CTLA-4 therapy, PD-1 inhibitors have relatively few severe irAEs (10-15\% vs. $30-40 \%$ grade $3-4$ toxicities) and are dose independent, with similar toxicities observed over a 100 -fold dosing range (16).

The most common irAEs are dermatitis, thyroiditis, colitis, pneumonitis, and arthritis. The incidence of irAEs vary not only between ICIs but also with primary malignancy, dosing regimen, and/or history of radiation therapy. Serious events are relatively uncommon but more clinically severe toxicities (Clinical Trials Common Adverse Event [CTCAE] grade 3-5) do occur in 10-15\% of patients; fatal events occur in approximately 1 in 300 patients (17). IrAEs can occur in virtually any organ system, most commonly during the first three months of therapy but may occur even up to six months after therapy discontinuation, and will likely be clinically classified as an "-itis" (eg, thyroiditis, colitis, pneumonitis) (18). Depending upon the severity of irAE(s), graded based upon the organ system(s) involved, the PD-1 inhibitor is held either temporarily or permanently and the patient may receive a course of steroids, which does not appear to dampen the anti-tumor effects (19). Despite 
these irAEs, the overall tolerability profile of the PD-1 inhibitors is generally favorable to that of traditional chemotherapy (20). However, these toxicity profiles have not undergone rigorous sub-group analysis regarding differences between age groups in the landmark trials ultimately leading to FDA-approval, and given their novelty, the long-term sequelae are not yet well defined. As older adults continue to make up larger portions of those receiving treatment in the "real world," it is prudent to enroll larger numbers of older adults into future prospective trials and analyze the existing, unpublished data for differences in toxicity profiles as this critical information will guide risk-benefit discussions and overall treatment strategies in this heterogeneous patient population (21). PD-1 inhibitors are changing oncology practice as they continue to show improved overall survival and progression free survival when compared to current standards of care across various cancers and patient demographics in large prospective clinical trials.

Despite the changes in immunobiology with age, the overall efficacy of PD-1 inhibitors in multiple settings has been reported to be comparable between older adults with cancer when compared to younger adults with cancer (22-24). However, when the sub-group analyses are scrutinized on an individual basis, there are trials with detectable differences in overall survival or progression free survival between analyzed age groups as well as trials that either lack sufficient enrollment to detect a difference or simply do not discuss it (Table 1). As with irAEs, future clinical trials should deem it a priority to enroll and carefully study as well as transparently report the outcomes of older adults with cancer as that data will mirror clinical applications and directly inform complex, bedside decision-making between the providers and patients. For more details, see "Specific considerations" below.

\section{PD-L1 inhibitors}

There are currently three FDA-approved PD-L1 inhibitors, atezolizumab (Tecentriq ${ }^{\circledR}$, Genentech), avelumab (Bavencio®, Pfizer) and durvalumab (Imfinzi®, AstraZeneca). Atezolizumab was the first in class to be approved in 2016 for both NSCLC and urothelial carcinoma. Avelumab was then approved in 2017 for both urothelial carcinoma and metastatic merkel cell carcinoma. Durvalumab was approved during 2017 in the setting of urothelial carcinoma followed by approval for NSCLC (stage III following chemoradiation) in 2018 (Table 1). All three are monoclonal antibodies directed against PD-L1, require intravenous infusion over 60 minutes, and are administered every two to three weeks.

As described, PD-L1 is typically expressed on APCs and facilitates self-tolerance. However, it is also expressed on tumors and promotes immune evasion. Similar to PD-1 inhibitors, PD-L1 inhibitors disrupt this signaling to promote tumor detection and destruction but come with a toxicity profile including irAEs. Most of the most common side effects when used as single agents are similar to PD-1 inhibitors; although avelumab has a somewhat unique side effect of infusion reactions (15-20\%, usually grade 1-2). PD-L1 inhibitor associated irAEs are also dose independent and can affect any organ system up to six months after treatment is discontinued requiring a similar treatment approach (16). These are also reported to be well-tolerated in comparison with traditional chemotherapy but require further study regarding frequency and severity in older adults $(20,21)$. While PD-L1 inhibitors were included in the reports demonstrating overall similar efficacy in multiple settings between 
different age groups, there is a relative paucity of data and further investigations are required to clearly delineate the risk-benefit ratio in treating older adults with PD-L1 inhibitors (2224).

\section{CTLA-4 inhibitor}

There is currently a single FDA-approved CTLA-4 inhibitor, ipilimumab (Yervoy®, BristolMyers Squibb). Ipilimumab was approved in 2011 by the FDA to treat metastatic melanoma, making it the first ICI available for clinical use. It went on to be approved for use in the adjuvant setting of melanoma in 2015 (Table 1). It is a monoclonal antibody directed against CTLA-4, requires intravenous infusion over 90 minutes, and is administered every three to 12 weeks.

CTLA-4 is a receptor found on T-cells and attenuates an immune response when bound to B7 on APCs, thus acting as an immune checkpoint. Ipilimumab binds to CTLA-4 and blocks its interaction with B7, allowing CD28 to instead bind B7 and promote T-cell activity. As discussed with PD-1 and PD-L1 inhibitors, bypassing an immune checkpoint to promote anti-tumor activities puts patients at risk for irAEs. Unlike PD-1 and PD-L1 inhibitors, the irAEs from ipilimumab occur more frequently and with a greater intensity as well as demonstrating dose-dependency (grade 3-5 irAEs occur in approximately 30-40\%, fatal events in approximately 1\%) (16). There is little known about how ipilimumab's irAEs and/or its clinical efficacy differ between age groups at this time, although it is commonly used across age groups.

\section{Combination Regimens}

As both PD-1/PD-L1 and CTLA-4 inhibitors function at distinct modes of T cell activation, combining these agents has mechanistic support and was hypothesized to enhance clinical activity. Nivolumab in combination with ipilimumab has been approved in three distinct settings, including metastatic colorectal cancer with microsatellite instability, metastatic melanoma, and renal cell carcinoma (Table 1). This combined regimen has been associated with higher response rates compared with anti-PD-1 monotherapy (eg, $60 \%$ vs. $\sim 45 \%$ for nivolumab monotherapy) (25). IrAEs occur with greater severity and frequency with combination therapy (grade 3-5 irAEs in 55-60\% of patients, fatal events $>1 \%$ ), requiring careful monitoring. As new combinations will undoubtedly be pursued, trials ought to include adequate numbers of older adults and assess for differences in efficacy and toxicity profiles.

ICIs are also being combined with other agents. Pembrolizumab (in combination with chemotherapy) and atezolizumab (in combination with chemotherapy and bevacizumab) have demonstrated improved survival in first-line NSCLC, and are now being routinely used. Both pembrolizumab and avelulmab combined with axitinib in metastatic renal cell carcinoma have also recently showed improved clinical outcomes $(26,27)$. A multitude of other clinical trials combining ICI with other standard and experimental therapies are now under development. 


\section{Non-ICI Immunotherapies}

In addition to the ICIs discussed, the armamentarium of immunotherapy continues to expand at a rapid pace. New targets within the realm of immune checkpoint disruption are now being pursued, including anti-CD47 antibodies that promote macrophage phagocytosis and subsequent T-cell activity (28). Chimeric antigen receptor (CAR) T-cell therapy continues to gain traction as it demonstrates clinical efficacy, though comes with unique side effect profiles including cytokine release syndrome and neurologic aberrations (29). In situ tumor infiltrating lymphocytes (TILs) are being explored as an upcoming immunotherapy with the idea that they can be harvested and then expanded in vitro for re-introduction and/or in combination with additional immunomodulators. Although many prior attempts at developing preventive cancer vaccines have been unsuccessful, new promising approaches, including personalized neoantigen-based vaccines are being tested $(30,31)$. Sipuleucel-T, a therapeutic cancer vaccine described as an "autologous cellular immunotherapy," is currently FDA-approved for metastatic castrate resistant prostate cancer (32). Bispecific Tcell Engagers (BiTEs) may be conceptualized as a dual-specific antibody, engaging T-cells with a pre-assigned target such as malignant B-Cells (33). Although direct cytokine therapy is growing obsolete in the setting of ICIs, intravesical Bacillus Calmette-Guerin continues to be used in the treatment superficial bladder cancers and works by inducing local immune upregulation. These tools, new and old, will pose additional challenges in treatment paradigms amongst older adults and require careful risk-benefit analysis for individual patients' clinical scenarios that will require intentionally designed clinical trials and analyses.

\section{Specific Considerations surrounding ICls in older patients}

Aging may impact the anti-tumor immune features in many ways. Older adults with cancer have a higher prevalence of comorbid conditions, functional impairments, and frailty which may alter the efficacy and tolerability of ICIs $(34,35)$. Moreover, changes associated with aging, or immunosenescence, comprise numerous and diverse well-described changes including thymic involution, increase in memory B cells and decreased hematopoiesis (36). Several studies have attempted to assess the differential efficacy and toxicity of ICI in older vs. younger patients. A number of clinical trials have done simple subgroup analyses between patients older vs. younger than 65 years. These studies have generally shown similar efficacy and toxicity, although many of these analyses are somewhat hampered since a large proportion of patients are clustered around 65 years (thus much of the comparison is between patients of similar age, e.g. a 64 vs. 66 year old patient). A recent retrospective study of older adults over 70 years of age with advanced stage lung cancer treated with ICIs, relatively high rates of ICI discontinuation, use of glucocorticoids, and hospitalizations were reported that appeared driven by performance status rather than chronological age or comorbid conditions (37). Case reports have shown that patients older than age 80 or even 90 may respond to therapy, although the rate of benefit is not clear (38). One large retrospective study in advanced melanoma patients reported similar benefits and toxicities in patients by decade (39). Two other retrospective studies even suggested that older patients may have superior benefits compared with their younger counterparts; this finding was supported by a more favorable antitumor balance of CD8 T cells / regulatory T cells in the 
tumor microenvironment $(40,41)$. One pre-clinical study, however, showed inferior efficacy in older mouse models, suggesting that age may have distinct effects on different tumor types and in various physiologic states (eg, frail vs. robust patients) (42). Meta-analyses of randomized trials suggested that while anti-PD-1 agents were effective in young vs. older patients, older patients had either equivalent or improved hazard ratio of death compared to control interventions, without enhanced toxicities $(22,43-45)$.

Regarding toxicities, studies have not shown enhanced toxicities in older patients. We observed, however, that patients with fatal toxicities appeared to be older than patients lacking these most severe toxicities (17). This suggests that while the incidence of these events overall may be similar, older patients may lack the functional reserve in some cases to recover from more severe events. Further, high-dose corticosteroids, the standard treatment of irAEs, may also be poorly tolerated in older patients with multiple co-morbidities (eg, diabetes, cardiac arrhythmias). Thus, while toxicity incidence is likely similar across age ranges, recovery from these events may be more challenging in older patients, particularly with limited functional status and comorbid conditions.

In our practice, we routinely offer ICI to older patients. We typically favor single agent antiPD-1 in metastatic melanoma, for example, as an alternative to more aggressive combination regimens in older and frail patients. However, we have certainly seen older patients with adverse prognostic features (from a cancer standpoint) benefit from combination therapy, and therapeutic selection should be discussed on a case by case basis. And after a long discussion elucidating patient preferences for treatment and the lack of available data, we at times also offer therapy to patients even with poor functional status, although clearly more work is needed to define the efficacy and tolerability of these agents in frail patients. Geriatric assessments may be helpful in determining which patients fail to benefit from these agents, and performance status is likely not sensitive enough to help stratify patients.

\section{Conclusions}

Given changing demographics in the US and worldwide, the majority of patients with cancer will be older adults (1). Many challenges exist in the treatment of older adults with ICI including the increased presence of comorbid conditions and functional impairments, along with metabolic changes and immunosenescence, that all complicate the use of these new treatments in older a patients (see figure 1). As novel immunotherapies and combinations are developed and are increasingly used for a variety of indications, how these therapies are tolerated and effective in older adults becomes increasingly important. As most clinical trial populations do not represent the real-world population, in terms of age distributions or presence of functional limitations, frailty, and comorbidities, more research is needed in these specific populations. Further understanding of the factors associated with treatment tolerability and response to new immunotherapy agents in older adults with cancer is necessary to inform treatment decision-making. How baseline factors, including functional limitations and the presence of geriatric syndromes such as frailty and sarcopenia, impact the tolerability and efficacy of these treatments is critically needed, but requires prospective evaluation. Lastly, how immunotherapies impact long-term health-related quality of life and 
the onset of functional and cognitive decline is needed, as these factors are frequently prioritized outcomes in older adults considering treatment (46).

\section{Acknowledgments}

Funding: Supported in part by the Walter B. Frommeyer Fellowship in Investigative Medicine at the University of Alabama at Birmingham (Williams) and the National Cancer Institute of the National Institutes of Health (K08CA234225 GRW, K23 CA204726 DBJ). The content is solely the responsibility of the authors and does not necessarily represent the official views of the National Institutes of Health. DBJ serves on advisory boards for Array Biopharma, BMS, Genoptix, Incyte, Merck, and Novartis and receives research support from BMS and Incyte.

\section{References}

1. Smith BD, Smith GL, Hurria A, Hortobagyi GN, Buchholz TA. Future of cancer incidence in the United States: burdens upon an aging, changing nation. Journal of clinical oncology : official journal of the American Society of Clinical Oncology. 2009;27(17):2758-65. [PubMed: 19403886]

2. Scher KS, Hurria A. Under-representation of older adults in cancer registration trials: known problem, little progress. Journal of clinical oncology : official journal of the American Society of Clinical Oncology. 2012;30(17):2036-8. [PubMed: 22547597]

*3. Mohile SG, Dale W, Somerfield MR, Schonberg MA, Boyd CM, Burhenn PS, et al. Practical Assessment and Management of Vulnerabilities in Older Patients Receiving Chemotherapy: ASCO Guideline for Geriatric Oncology. Journal of clinical oncology : official journal of the American Society of Clinical Oncology. 2018:JCO2018788687.(Recent ASCO guidelines on the use of geriatric assessment in the management of older adults in oncology)

4. Jolly TA, Deal AM, Nyrop KA, Williams GR, Pergolotti M, Wood WA, et al. Geriatric assessmentidentified deficits in older cancer patients with normal performance status. The oncologist. 2015;20(4):379-85. [PubMed: 25765876]

5. Kimmick GG, Fleming R, Muss HB, Balducci L. Cancer chemotherapy in older adults. A tolerability perspective. Drugs \& aging. 1997;10(1):34-49. [PubMed: 9111706]

6. Balducci L, Yates J. General guidelines for the management of older patients with cancer. Oncology (Williston Park). 2000;14(11A):221-7. [PubMed: 11195414]

7. Williams GR, Rier HN, McDonald A, Shachar SS. Sarcopenia \& aging in cancer. Journal of geriatric oncology. 2018.

8. Nishijima TF, Deal AM, Williams GR, Sanoff HK, Nyrop KA, Muss HB. Chemotherapy Toxicity Risk Score for Treatment Decisions in Older Adults with Advanced Solid Tumors. The oncologist. 2018.

9. Hurria A, Togawa K, Mohile SG, Owusu C, Klepin HD, Gross CP, et al. Predicting chemotherapy toxicity in older adults with cancer: a prospective multicenter study. Journal of clinical oncology : official journal of the American Society of Clinical Oncology. 2011;29(25):3457-65. [PubMed: 21810685]

10. Williams GR. Geriatric Assessment: Precision Medicine for Older Adults With Cancer. Journal of oncology practice / American Society of Clinical Oncology. 2018;14(2):97-8.

11. Helissey C, Vicier C, Champiat S. The development of immunotherapy in older adults: New treatments, new toxicities? Journal of geriatric oncology. 2016;7(5):325-33. [PubMed: 27318796]

12. Murray JM, Kaufmann GR, Hodgkin PD, Lewin SR, Kelleher AD, Davenport MP, et al. Naive T cells are maintained by thymic output in early ages but by proliferation without phenotypic change after age twenty. Immunol Cell Biol. 2003;81(6):487-95. [PubMed: 14636246]

13. Nikolich-Zugich $\mathbf{J}$ Aging of the T cell compartment in mice and humans: from no naive expectations to foggy memories. J Immunol. 2014;193(6):2622-9. [PubMed: 25193936]

14. Nikolich-Zugich $\mathrm{J}$ The twilight of immunity: emerging concepts in aging of the immune system. Nat Immunol. 2018;19(1):10-9. [PubMed: 29242543] 
15. Palmer S, Albergante L, Blackburn CC, Newman TJ. Thymic involution and rising disease incidence with age. Proceedings of the National Academy of Sciences of the United States of America. 2018;115(8):1883-8. [PubMed: 29432166]

*16. Johnson DB, Chandra S, Sosman JA. Immune Checkpoint Inhibitor Toxicity in 2018. JAMA : the journal of the American Medical Association. 2018;320(16):1702-3. [PubMed: 30286224] (JAMA insight review on the clinical presentation and management of the most common and severe immune check point toxicities that nonconologists are likely to encounter)

17. Wang DY, Salem JE, Cohen JV, Chandra S, Menzer C, Ye F, et al. Fatal Toxic Effects Associated With Immune Checkpoint Inhibitors: A Systematic Review and Meta-analysis. JAMA Oncol. 2018;4(12):1721-8. [PubMed: 30242316]

18. Postow MA, Sidlow R, Hellmann MD. Immune-Related Adverse Events Associated with Immune Checkpoint Blockade. The New England journal of medicine. 2018;378(2):158-68. [PubMed: 29320654]

19. Weber JS, Hodi FS, Wolchok JD, Topalian SL, Schadendorf D, Larkin J, et al. Safety Profile of Nivolumab Monotherapy: A Pooled Analysis of Patients With Advanced Melanoma. Journal of clinical oncology : official journal of the American Society of Clinical Oncology. 2017;35(7):78592. [PubMed: 28068177]

*20. Nishijima TF, Shachar SS, Nyrop KA, Muss HB. Safety and Tolerability of PD-1/PD-L1 Inhibitors Compared with Chemotherapy in Patients with Advanced Cancer: A Meta-Analysis. The oncologist. 2017;22(4):470-9. [PubMed: 28275115] (Systematic review and meta-analyses that demonstrates that PD1/PD-L1 inhibitors are overall better tolerated than chemotherapy)

21. Kanesvaran R, Cordoba R, Maggiore R. Immunotherapy in Older Adults With Advanced Cancers: Implications for Clinical Decision-Making and Future Research. American Society of Clinical Oncology educational book / ASCO American Society of Clinical Oncology Meeting. 2018(38): 400-14.

22. Elias R, Giobbie-Hurder A, McCleary NJ, Ott P, Hodi FS, Rahma O. Efficacy of PD-1 \& PD-L1 inhibitors in older adults: a meta-analysis. J Immunother Cancer. 2018;6(1):26. [PubMed: 29618381]

23. Elias R, Hartshorn K, Rahma O, Lin N, Snyder-Cappione JE. Aging,immune senescence, and immunotherapy:A comprehensive review. Seminars in oncology. 2018;45(4):187-200. [PubMed: 30539714]

24. Elias R, Morales J, Rehman Y, Khurshid H. Immune Checkpoint Inhibitors in Older Adults. Curr Oncol Rep. 2016;18(8):47. [PubMed: 27287329]

25. Wolchok JD, Chiarion-Sileni V, Gonzalez R, Rutkowski P, Grob JJ, Cowey CL, et al. Overall Survival with Combined Nivolumab and Ipilimumab in Advanced Melanoma. The New England journal of medicine. 2017;377(14):1345-56. [PubMed: 28889792]

26. Atkins MB, Plimack ER, Puzanov I, Fishman MN, McDermott DF, Cho DC, et al. Axitinib in combination with pembrolizumab in patients with advanced renal cell cancer: a non-randomised, open-label, dose-finding, and dose-expansion phase 1b trial. The lancet oncology. 2018;19(3):40515. [PubMed: 29439857]

27. Choueiri TK, Larkin J, Oya M, Thistlethwaite F, Martignoni M, Nathan P, et al. Preliminary results for avelumab plus axitinib as first-line therapy in patients with advanced clear-cell renal-cell carcinoma (JAVELIN Renal 100): an open-label, dose-finding and dose-expansion, phase $1 \mathrm{~b}$ trial. The lancet oncology. 2018;19(4):451-60. [PubMed: 29530667]

28. Advani R, Flinn I, Popplewell L, Forero A, Bartlett NL, Ghosh N, et al. CD47 Blockade by Hu5F9-G4 and Rituximab in Non-Hodgkin's Lymphoma. The New England journal of medicine. 2018;379(18):1711-21. [PubMed: 30380386]

29. Neelapu SS, Locke FL, Go WY. CAR T-Cell Therapy in Large B-Cell Lymphoma. The New England journal of medicine. 2018;378(11):1065.

30. Ott PA, Hu Z, Keskin DB, Shukla SA, Sun J, Bozym DJ, et al. An immunogenic personal neoantigen vaccine for patients with melanoma. Nature. 2017;547(7662):217-21. [PubMed: 28678778] 
31. Sahin U, Derhovanessian E, Miller M, Kloke BP, Simon P, Lower M, et al. Personalized RNA mutanome vaccines mobilize poly-specific therapeutic immunity against cancer. Nature. 2017;547(7662):222-6. [PubMed: 28678784]

32. Kantoff PW, Higano CS, Shore ND, Berger ER, Small EJ, Penson DF, et al. Sipuleucel-T immunotherapy for castration-resistant prostate cancer. The New England journal of medicine. 2010;363(5):411-22. [PubMed: 20818862]

33. Kantarjian H, Jabbour E, Topp MS. Blinatumomab for Acute Lymphoblastic Leukemia. The New England journal of medicine. 2017;376(23):e49.

34. Guerard EJ, Deal AM, Chang Y, Williams GR, Nyrop KA, Pergolotti M, et al. Frailty Index Developed From a Cancer-Specific Geriatric Assessment and the Association With Mortality Among Older Adults With Cancer. Journal of the National Comprehensive Cancer Network : JNCCN. 2017;15(7):894-902. [PubMed: 28687577]

35. Williams GR, Mackenzie A, Magnuson A, Olin R, Chapman A, Mohile S, et al. Comorbidity in older adults with cancer. Journal of geriatric oncology. 2016;7(4):249-57. [PubMed: 26725537]

36. Pawelec G Does patient age influence anti-cancer immunity? Semin Immunopathol. 2019;41(1): 125-31. [PubMed: 30006738]

37. Muchnik E, Loh KP, Strawderman M, Magnuson A, Mohile SG, Estrah V, et al. Immune Checkpoint Inhibitors in Real-World Treatment of Older Adults with Non-Small Cell Lung Cancer. Journal of the American Geriatrics Society. 2019.

38. Johnpulle RA, Conry RM, Sosman JA, Puzanov I, Johnson DB. Responses to immune checkpoint inhibitors in nonagenarians. Oncoimmunology. 2016;5(11):e1234572. [PubMed: 27999751]

39. Betof AS, Nipp RD, Giobbie-Hurder A, Johnpulle RAN, Rubin K, Rubinstein SM, et al. Impact of Age on Outcomes with Immunotherapy for Patients with Melanoma. The oncologist. 2017;22(8): 963-71. [PubMed: 28476944]

40. Ben-Betzalel G, Steinberg-Silman Y, Stoff R, Asher N, Shapira-Frommer R, Schachter J, et al. Immunotherapy comes of age in octagenarian and nonagenarian metastatic melanoma patients. Eur J Cancer. 2019;108:61-8. [PubMed: 30648631]

41. Kugel CH 3rd, Douglass SM, Webster MR, Kaur A, Liu Q, Yin X, et al. Age Correlates with Response to Anti-PD1, Reflecting Age-Related Differences in Intratumoral Effector and Regulatory T-Cell Populations. Clinical cancer research : an official journal of the American Association for Cancer Research. 2018;24(21):5347-56. [PubMed: 29898988]

42. Padron A, Hurez V, Gupta HB, Clark CA, Pandeswara SL, Yuan B, et al. Age effects of distinct immune checkpoint blockade treatments in a mouse melanoma model. Experimental gerontology. 2018;105:146-54. [PubMed: 29326088]

43. Li P, Yang X, Feng Y, Wu L, Ma W, Ding G, et al. The impact of immunosenescence on the efficacy of immune checkpoint inhibitors in melanoma patients: a meta-analysis. Onco Targets Ther. 2018;11:7521-7. [PubMed: 30464500]

44. Marur S, Singh H, Mishra-Kalyani P, Larkins E, Keegan P, Sridhara R, et al. FDA analyses of survival in older adults with metastatic non-small cell lung cancer in controlled trials of PD-1/PDL1 blocking antibodies. Seminars in oncology. 2018;45(4):220-5. [PubMed: 30391014]

*45. Nishijima TF, Muss HB, Shachar SS, Moschos SJ. Comparison of efficacy of immune checkpoint inhibitors (ICIs) between younger and older patients: A systematic review and meta-analysis. Cancer treatment reviews. 2016;45:30-7. [PubMed: 26946217] (Systematic review and metaanalysis that demonstrates a similar benefit in overall survival between older and younger patients treated with immune checkpoint inhibitors)

46. Fried TR, Bradley EH, Towle VR, Allore H. Understanding the treatment preferences of seriously ill patients. The New England journal of medicine. 2002;346(14):1061-6. [PubMed: 11932474] 


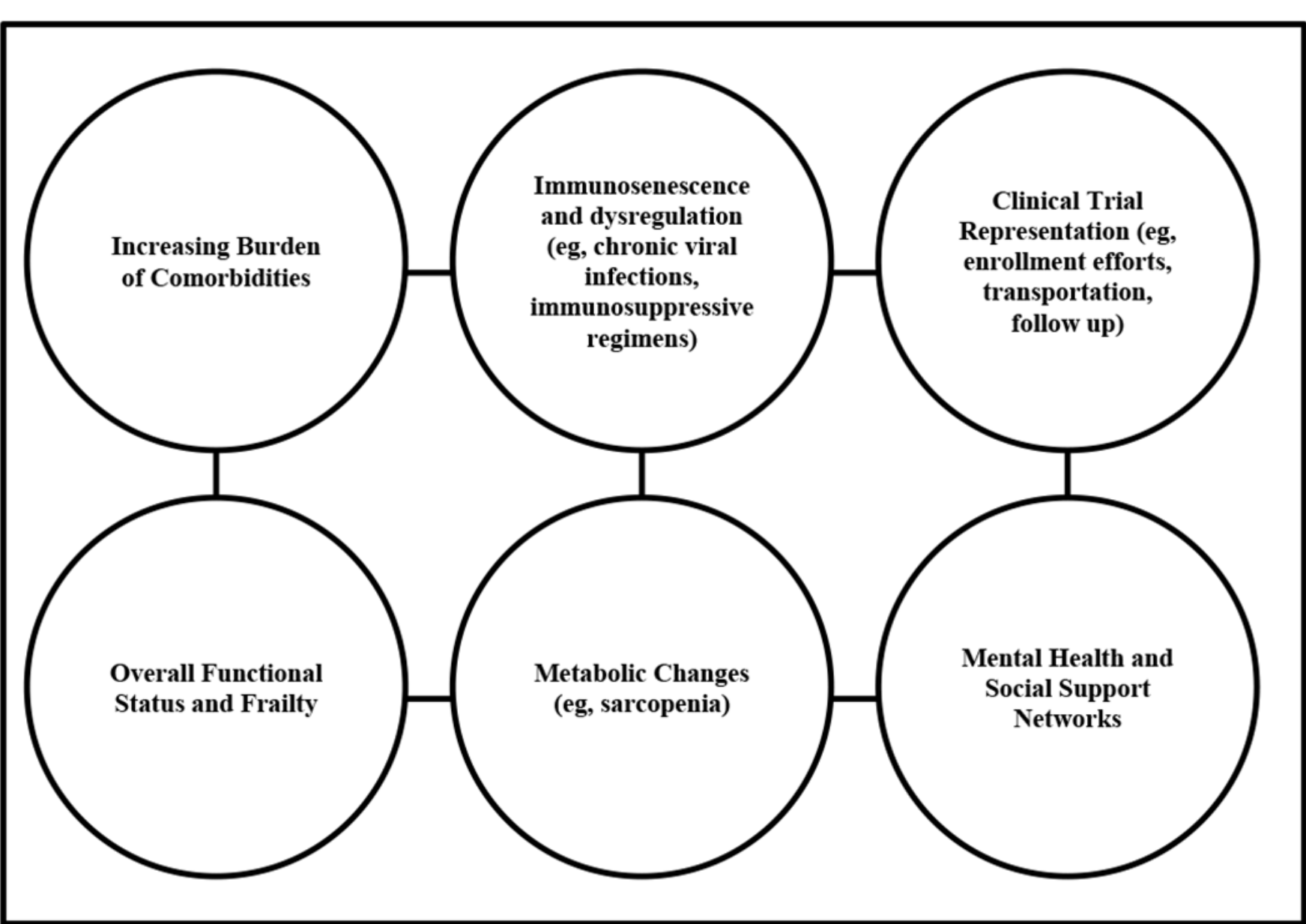

Figure 1.

Challenges with Treating Older Adults and Immunotherapy 

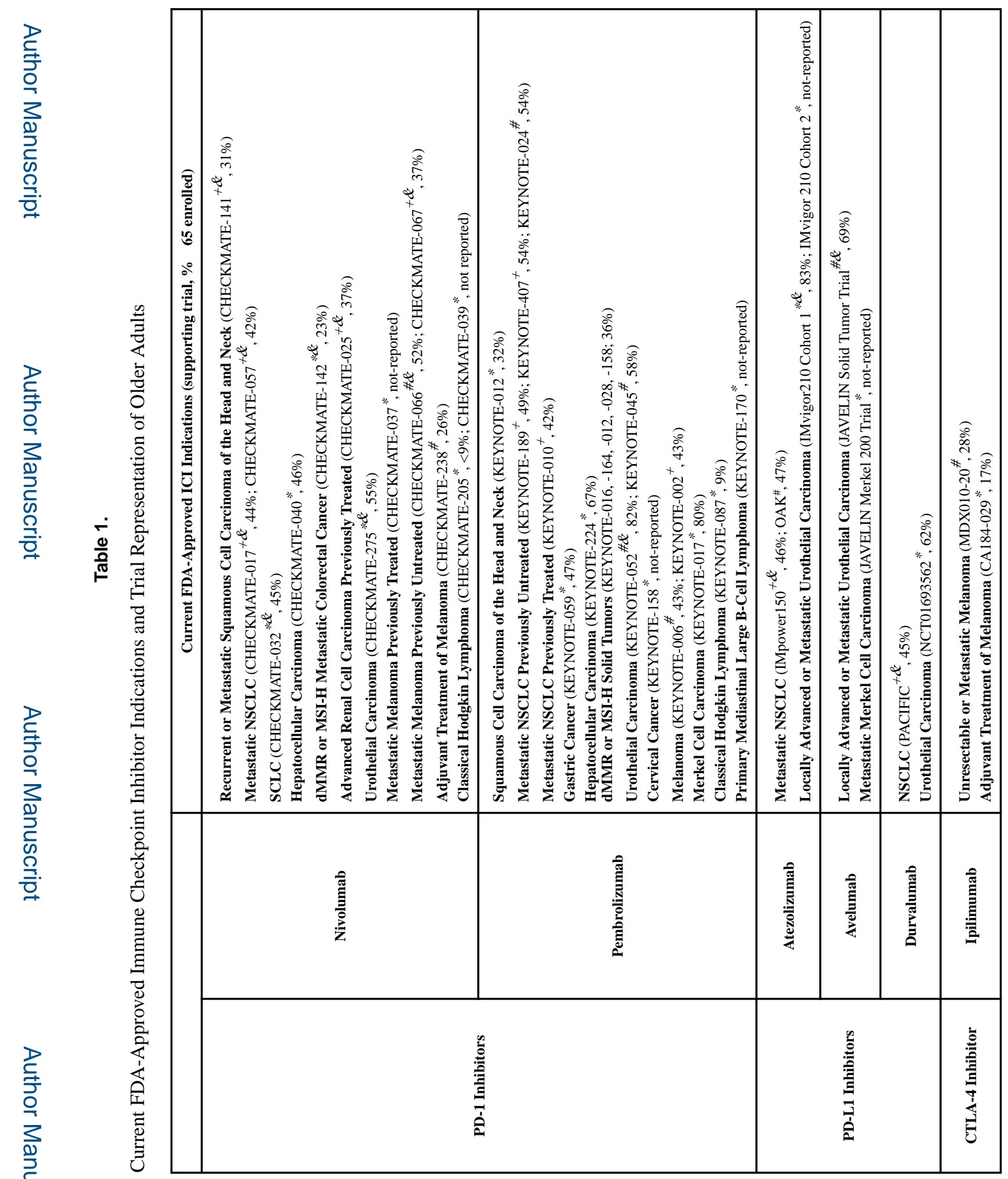

Curr Oncol Rep. Author manuscript; available in PMC 2020 May 07. 


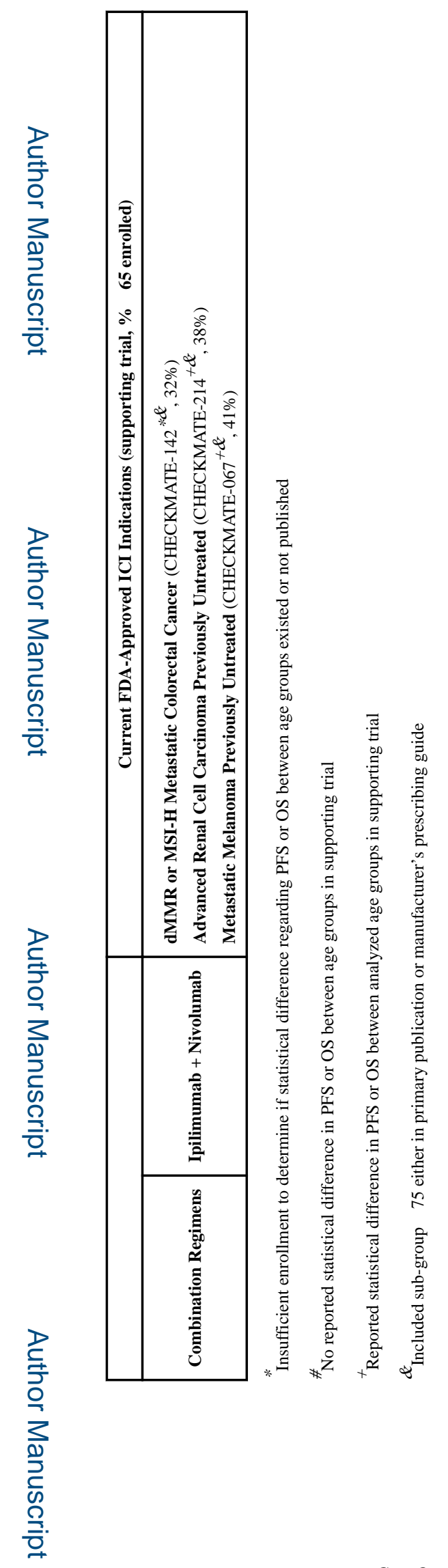

Curr Oncol Rep. Author manuscript; available in PMC 2020 May 07. 


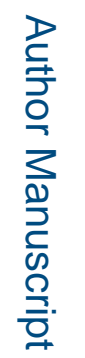

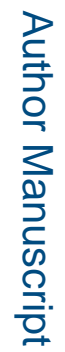

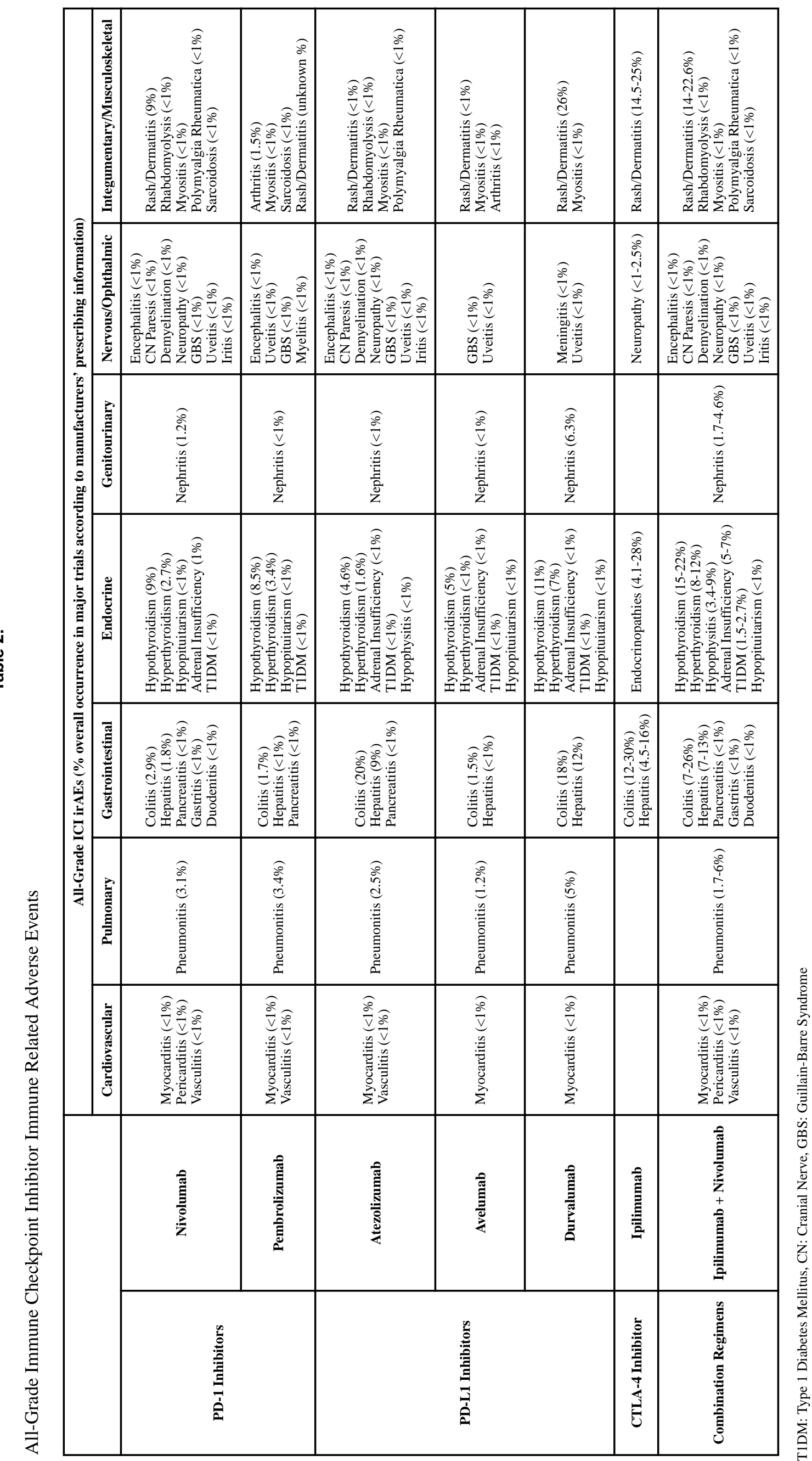

